\title{
Flight Stability of an Asymmetric Projectile with Activating Canards
}

\author{
Gene Cooper* and Frank Fresconi ${ }^{\ddagger}$ \\ U.S. Army Research Laboratory, Aberdeen Proving Ground, Maryland 2101 \\ and \\ Mark Costello \\ Georgia Institute of Technology, Atlanta, Georgia 30332 \\ DOI: $10.2514 / 1 . \mathrm{A} 32022$
}

\begin{abstract}
Driven by the creation of new smart projectile concepts with maneuver capability, projectile configurations with large aerodynamic asymmetries are becoming more common. Standard linear stability theory for projectiles assumes the projectile is symmetric, both from aerodynamic and mass properties perspectives. The work reported here extends standard projectile linear theory to account for aerodynamic asymmetries caused by actuating canards. Differences between standard linear and extended linear theories reported here are highlighted. To validate the theory, time simulation of the extended linear theory and a fully nonlinear trajectory simulation are made for a representative scenario, with excellent agreement noted. The extended linear-projectile theory offers a tool to address flight stability of projectiles with aerodynamic configuration asymmetries.
\end{abstract}

\section{Nomenclature}

$C_{i} \quad=$ projectile aerodynamic coefficients

$C_{i c} \quad=$ projectile aerodynamic coefficients due to a canard

$D=$ projectile characteristic length (diameter), $\mathrm{ft}$

$f, \theta, \psi=$ Euler roll, pitch, and yaw angles of projectile

$g \quad=$ gravitational constant, $\mathrm{ft} \cdot \mathrm{s}^{-2}$

$\mathbf{I}_{n}, \mathbf{J}_{n}, \mathbf{K}_{n}=$ unit vectors for a coordinate system designated by $n$

$I_{X}, I_{T} \quad=$ mass moments of inertia, slug $\cdot \mathrm{ft}^{2}$

$m=$ projectile mass, slug

$p, q, r=$ angular velocity components vector of projectile, $\mathrm{s}^{-1}$

$r \quad=$ atmosphere density, slug $\cdot \mathrm{ft}^{-3}$

$S, \bar{S} \quad=$ complex and conjugate projectile yaw rates

$\mathrm{SL}_{\mathrm{Cg}} \quad=$ station-line center of gravity, $\mathrm{ft}$

$\mathrm{SL}_{\mathrm{COP}}=$ station-line center of pressure, $\mathrm{ft}$

$\mathrm{SL}_{\mathrm{COPC}}=$ station-line center of pressure of canard, $\mathrm{ft}$

$\mathrm{SL}_{\text {MAG }}=$ station-line center of Magnus, $\mathrm{ft}$

$u, v, w \quad=$ mass center velocity components in the body reference frame, $\mathrm{ft} \cdot \mathrm{s}^{-1}$

$\left|\mathbf{V}_{\ddot{A} / I}\right|=V=$ projectile center-of-mass velocity, $\mathrm{ft} \cdot \mathrm{s}^{-1}$

$\Delta x c, \Delta y c=$ displacement of canard center of pressure relative to mass center, $\mathrm{ft}$

$\delta=$ canard deflection angle with respect to projectile axis
Presented as Paper 2010-7636 at the AIAA Guidance, Navigation, and Control, Atmospheric Flight Mechanics, Modeling and Simulation Technologies, AIAA/AAS Astrodynamics Specialist, and Atmospheric and Space Environments Conferences, Toronto, 2-5 August 2010; received 20 December 2010; revision received 26 April 2011; accepted for publication 19 May 2011. This material is declared a work of the U.S. Government and is not subject to copyright protection in the United States. Copies of this paper may be made for personal or internal use, on condition that the copier pay the $\$ 10.00$ per-copy fee to the Copyright Clearance Center, Inc., 222 Rosewood Drive, Danvers, MA 01923; include the code 0022-4650/12 and \$10.00 in correspondence with the CCC.

*Research Physicist. Senior Member AIAA.

${ }^{\dagger}$ Mechanical Engineer. Member AIAA.

${ }^{\ddagger}$ Sikorsky Associate Professor, School of Aerospace Engineering. Associate Fellow AIAA.

\section{Introduction}

$\mathbf{T}$ HERE is no doubt that there are many ways to create controllable forces and moments on a projectile to enable sufficiently large changes to its trajectory for flight control purposes. A traditional and powerful way to control a projectile is through aerodynamic force and moment changes. Examples of the type of mechanism include canards [1]. Since the flight control system usually commands an inertial position change, the control mechanism fixed on the body will oscillate at the roll rate of the projectile. Many aerodynamic control mechanisms (like canards) introduce an asymmetry into the basic configuration.

Projectile linear theory has long been an analytical workhorse in the ballistics community. Over time, projectile linear theory has been used for stability analysis, aerodynamic coefficient estimation using range data, and fast trajectory prediction. Basic projectile linear theory has been extended by various authors to handle more sophisticated aerodynamic models [2] , asymmetric configurations including mass properties $[\underline{3}, \underline{4}]$, fluid payloads $[\underline{5}, \underline{6}]$, moving internal parts [7-9], dual spin projectiles [10,11], ascending and descending flight $[\overline{12}]$, and lateral force impulses [13-16]. The work reported here is along these lines and develops an extended linear theory applicable to aerodynamically asymmetric projectile configurations. Moreover, the paper focuses on projectiles with dithering canards similar to that shown in Fig. 1. The extended linear theory is validated against nonlinear six-degree-of-freedom (6-DOF) calculations for an example projectile.

The paper begins with a description of the basic projectile mathematical model followed by the development of the extended linear theory. The theory is then applied to an example configuration.

\section{Trajectory Equations of Motion}

To facilitate the mathematical description of the dynamic model, we define the vector component operator $\mathbf{C}_{\mathbf{n}}$ and the vector-product operator $\mathbf{S}$ as a skew symmetric matrix applied to any vector $\mathbf{A}$ as

$$
\begin{gathered}
\mathbf{A}=\mathbf{I}_{n} A_{x}+\mathbf{J}_{n} A_{y}+\mathbf{K}_{n} A_{z} \quad C_{n}(\mathbf{A}) \equiv\left\{\begin{array}{l}
A_{x} \\
A_{y} \\
A_{z}
\end{array}\right\} \\
S(\mathbf{A}) \equiv\left[\begin{array}{ccc}
0 & -A_{z} & A_{y} \\
A_{z} & 0 & -A_{x} \\
-A_{y} & A_{x} & 0
\end{array}\right]
\end{gathered}
$$




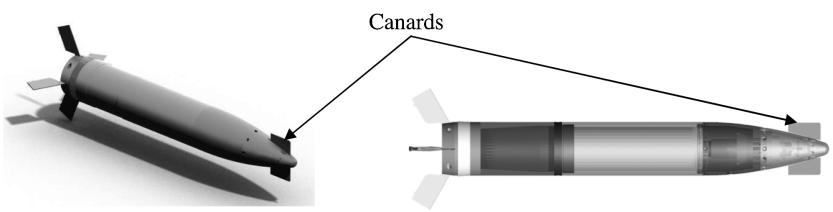

Fig. 1 Asymmetric airframe with or without control mechanism.

These definitions provide a succinct method of writing the mathematics in this paper. Also, the standard shorthand for sine and cosine are employed: $\sin (\alpha)=s_{\alpha}$ and $\cos (\alpha)=c_{\alpha}$.

Placing two canards, diametrically positioned on a symmetric projectile, results in additional aeroloads that must be accounted for in the equations of motion. For this study, two canards are located at projectile roll angles $\varphi=0$ and $\varphi=\pi$; see Fig. 1 . This angular separation $\Delta \phi>2 \pi / 3$ introduces rotational asymmetry [3]; therefore, it is preferable to write the dynamic equations in the body reference frame. In fact, the analysis is more tractable if we work in the body frame instead of the well-known nonrolling frame, particularly if the asymmetry is fluctuating. The angular velocity of the body frame is

$$
\boldsymbol{\omega}_{B / I}=p \mathbf{I}_{B}+q \mathbf{J}_{B}+r \mathbf{K}_{B}
$$

The equations of motion relative to the inertial and body frames are

$$
C_{I}\left(\dot{\mathbf{V}}_{A / I}\right)=\left[\begin{array}{ccc}
c_{\theta} c_{\psi} & s_{\phi} s_{\theta} c_{\psi}-c_{\phi} s_{\psi} & c_{\phi} s_{\theta} c_{\psi}+s_{\phi} s_{\psi} \\
c_{\theta} s_{\psi} & s_{\phi} s_{\theta} s_{\psi}+c_{\phi} c_{\psi} & c_{\phi} s_{\theta} s_{\psi}-s_{\phi} c_{\psi} \\
-s_{\theta} & s_{\phi} c_{\theta} & c_{\phi} c_{\theta}
\end{array}\right] C_{B}\left(\dot{\mathbf{V}}_{A / I}\right)
$$

$$
\left\{\begin{array}{c}
\dot{\phi} \\
\dot{\theta} \\
\dot{\psi}
\end{array}\right\}=\left[\begin{array}{ccc}
1 & s_{\phi} t_{\theta} & c_{\phi} t_{\theta} \\
0 & c_{\phi} & -s_{\phi} \\
0 & s_{\phi} / c_{\theta} & c_{\phi} / c_{\theta}
\end{array}\right] C_{B}\left(\omega_{B / I}\right)
$$

$$
\begin{gathered}
C_{B}\left(\dot{\mathbf{V}}_{\otimes / I}\right)=\frac{1}{m} C_{B}(\mathbf{F})-S\left(\omega_{B / I}\right) C_{B}\left(\mathbf{V}_{\otimes / I}\right) \\
C_{B}\left(\dot{\omega}_{B / I}\right)=[I]^{-1}\left(C_{B}(\mathbf{M})-S\left(\omega_{B / I}\right)[I] C_{B}\left(\omega_{B / I}\right)\right)
\end{gathered}
$$

The force acting on the projectile in Eq. (6) comprises the weight force $(W)$, the aerodynamic force, and the canard force $(C)$. The aerodynamic force is split into a standard $(A)$ and Magnus $(M)$ force. The combination of forces is expressed in Eq. (7):

$$
C_{B}(\mathbf{F})=C_{B}\left(\mathbf{F}_{W}\right)+C_{B}\left(\mathbf{F}_{A}\right)+C_{B}\left(\mathbf{F}_{M}\right)+C_{B}\left(\mathbf{F}_{C}\right)
$$

Equation (8) gives the weight force in the body coordinate system:

$$
C_{B}\left(\mathbf{F}_{W}\right)=m g\left\{\begin{array}{l}
-s_{\theta} \\
s_{\phi} c_{\theta} \\
c_{\phi} c_{\theta}
\end{array}\right\}
$$

Equation (9) provides the expression for the aerodynamic force that acts upon the projectile at the aerodynamic center of pressure:

$$
C_{B}\left(\mathbf{F}_{A}\right)=-\frac{\pi}{8} \rho V^{2} D^{2}\left\{\begin{array}{c}
C_{X 0}+C_{X 2}\left(v^{2}+w^{2}\right) / V^{2} \\
C_{Y 0}+C_{N A} v / V \\
C_{Z 0}+C_{N A} w / V
\end{array}\right\}
$$

Equation (10) expresses the Magnus force acting on the projectile at the Magnus force center:

$$
C_{B}\left(\mathbf{F}_{M}\right)=\frac{\pi}{8} \rho V^{2} D^{2}\left\{\begin{array}{c}
0 \\
\frac{p D C_{N P A} w}{2 V^{2}} \\
\frac{-p D C_{N P A} v}{2 V^{2}}
\end{array}\right\}
$$

The projectile forward velocity is given in Eq. (11):

$$
V=\sqrt{u^{2}+v^{2}+w^{2}}
$$

The moment acting on the projectile in Eq. (6) comprises the moment due to the standard aerodynamic force $(A)$, the moment due to the Magnus aerodynamic force $(M)$, the unsteady aerodynamic moment $(U A)$, and the canard moment $(C)$, as shown in Eq. (12):

$$
C_{B}(\mathbf{M})=C_{B}\left(\mathbf{M}_{A}\right)+C_{B}\left(\mathbf{M}_{M}\right)+C_{B}\left(\mathbf{M}_{U A}\right)+C_{B}\left(\mathbf{M}_{C}\right)
$$

The moments due to the aerodynamic and Magnus forces are expressed in Eq. (13):

$$
C_{B}\left(\mathbf{M}_{A}\right)=S\left(\mathbf{R}_{\otimes \rightarrow C p}\right) C_{B}\left(\mathbf{F}_{A}\right) \quad C_{B}\left(\mathbf{M}_{M}\right)=S\left(\mathbf{R}_{\otimes \rightarrow C M}\right) C_{B}\left(\mathbf{F}_{M}\right)
$$

The unsteady aerodynamic moments acting on the projectile are expressed in Eq. (14):

$$
C_{B}\left(\mathbf{M}_{U A}\right)=\frac{\pi}{8} \rho V^{2} D^{3}\left\{\begin{array}{c}
C_{D D}+\frac{p D C_{L P}}{2 V} \\
\frac{q D C_{M Q}}{2 V} \\
\frac{r D C_{M Q}}{2 V}
\end{array}\right\}
$$

The coefficients used in this aerodynamic model are specific functions of the projectile Mach number. For fin-stabilized projectiles, the classic Magnus force and moment associated with spinstabilized projectiles are typically ignored since its effect is rather small for slowly rolling projectiles. A Magnus moment with physical mechanisms specific to fin-stabilized projectiles (more properly expressed as a dynamic side moment due to spin and angle of attack), however, may exist [17]. Expressions for the canard force $X_{B}\left(\mathbf{F}_{C}\right)$ and canard moment $\overline{X_{B}}\left(\mathbf{M}_{C}\right)$ are derived in the following.

\section{Canard Force and Moment Model}

Consider the change in the aerodynamic loads on a projectile when the pitch angle of a canard lifting surface is deflected. Figure 2 has a schematic of the canard configuration.

This analysis is for two canards located at projectile roll angles $\varphi=0$ and $\varphi=\pi$ such that their respective centers of pressures are

$$
C_{B}\left(\mathbf{R}_{\otimes \rightarrow C 1}\right)=\left\{\begin{array}{c}
\Delta x c \\
\Delta y c \\
0
\end{array}\right\}, \quad C_{B}\left(\mathbf{R}_{\otimes \rightarrow C 2}\right)=\left\{\begin{array}{c}
\Delta x c \\
-\Delta y c \\
0
\end{array}\right\}
$$

The relative aerodynamic velocities of the two canard lifting surfaces are calculate from

$$
\begin{aligned}
& C_{B}\left(\mathbf{V}_{C 1 / I}\right)=C_{B}\left(\mathbf{V}_{\otimes / I}\right)+S\left(\omega_{B / I}\right) C_{B}\left(\mathbf{r}_{\otimes \rightarrow C 1}\right) \\
& C_{B}\left(\mathbf{V}_{C 2 / I}\right)=C_{B}\left(\mathbf{V}_{\otimes / I}\right)+S\left(\omega_{B / I}\right) C_{B}\left(\mathbf{r}_{\otimes \rightarrow C 2}\right)
\end{aligned}
$$

Now, generate the coordinate transforms from body coordinates of the canard coordinates with deflection angle $\delta$ :

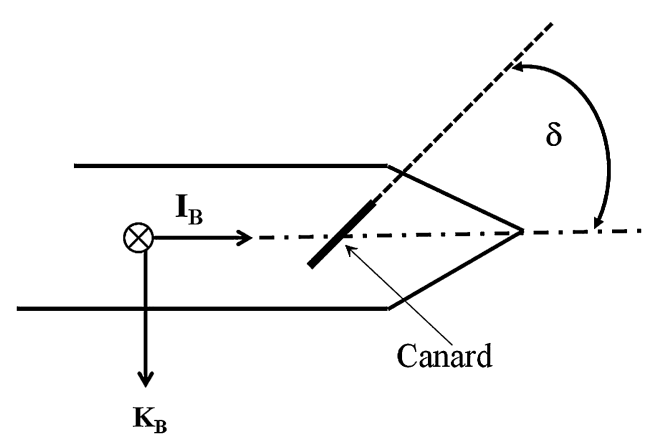

Fig. 2 Configuration of projectile with canard. 


$$
\begin{aligned}
& \left\{\begin{array}{c}
\mathbf{I}_{c 1} \\
\mathbf{J}_{c 1} \\
\mathbf{K}_{c 1}
\end{array}\right\}=T_{1}\left\{\begin{array}{l}
\mathbf{I}_{B} \\
\mathbf{J}_{B} \\
\mathbf{K}_{B}
\end{array}\right\}=\left[\begin{array}{ccc}
c_{\delta} & 0 & -s_{\delta} \\
0 & 1 & 0 \\
s_{\delta} & 0 & c_{\delta}
\end{array}\right]\left\{\begin{array}{l}
\mathbf{I}_{B} \\
\mathbf{J}_{B} \\
\mathbf{K}_{B}
\end{array}\right\} ; \quad \phi=0 \\
& \left\{\begin{array}{c}
\mathbf{I}_{c 2} \\
\mathbf{J}_{c 2} \\
\mathbf{K}_{c 2}
\end{array}\right\}=T_{2}\left\{\begin{array}{c}
\mathbf{I}_{B} \\
\mathbf{J}_{B} \\
\mathbf{K}_{B}
\end{array}\right\} \\
& =\left[\begin{array}{ccc}
c_{\delta} & 0 & -s_{\delta} \\
0 & 1 & 0 \\
s_{\delta} & 0 & c_{\delta}
\end{array}\right]\left[\begin{array}{ccc}
1 & 0 & 0 \\
0 & -1 & 0 \\
0 & 0 & -1
\end{array}\right]\left\{\begin{array}{c}
\mathbf{I}_{B} \\
\mathbf{J}_{B} \\
\mathbf{K}_{B}
\end{array}\right\} ; \quad \phi=\pi
\end{aligned}
$$

Therefore, the relative velocities of the lifting surfaces written in local canard coordinates have the form

$$
C_{C 1}\left(\mathbf{V}_{C 1 / I}\right)=T_{1} C_{B}\left(\mathbf{V}_{C 1 / I}\right), \quad C_{C 2}\left(\mathbf{V}_{C 2 / I}\right)=T_{2} C_{B}\left(\mathbf{V}_{C 2 / I}\right)
$$

Construct the angle of attack for each canard $\alpha_{j}$ as

$$
\begin{aligned}
& \tan \left(\alpha_{1}\right)=\frac{\mathbf{K}_{C 1} \bullet C_{C 1}\left(\mathbf{V}_{C 1 / I}\right)}{\|\left(1-I_{C 1} \bullet C_{C 1}\left(V_{C 1 / I}\right) \|\right.} \\
& \tan \left(\alpha_{2}\right)=\frac{\mathbf{K}_{C 2} \bullet C_{C 2}\left(\mathbf{V}_{C 2 / I}\right)}{\|\left(1-\mathbf{I}_{C 2} \bullet C_{C 2}\left(\mathbf{V}_{C 2 / I}\right) \|\right.}
\end{aligned}
$$

The axial $X_{j}$ and normal $N_{j}$ aerodynamic forces acting on the lifting surfaces of the canards are modeled as

$$
C_{C j}\left(\mathbf{F}_{A j}\right) \equiv\left\{\begin{array}{c}
X_{j} \\
0 \\
N_{j}
\end{array}\right\}=\frac{\pi}{8} \rho\left|C_{B}\left(\mathbf{V}_{C 1 / I}\right)\right|^{2} D^{2}\left\{\begin{array}{c}
C_{X 0 C}+C_{X 2 C} \alpha_{j}^{2} \\
0 \\
C_{N A C} \alpha_{j}
\end{array}\right\}
$$

Thus, the force $\mathbf{F}_{C}$ and moment $\mathbf{M}_{C}$ due to the two canards take the form

$$
\begin{gathered}
C_{B}\left(\mathbf{F}_{C}\right)=T_{1}^{-1} C_{C 1}\left(-\mathbf{F}_{A 1}\right)+T_{2}^{-1} C_{C 2}\left(-\mathbf{F}_{A 2}\right) \\
C_{B}\left(\mathbf{M}_{C}\right)=S\left(r_{\otimes \rightarrow C 1}\right) T_{1}^{-1} C_{C 1}\left(-\mathbf{F}_{A 1}\right)+S\left(r_{\otimes \rightarrow C 2}\right) T_{2}^{-1} C_{C 2}\left(-\mathbf{F}_{A 2}\right)
\end{gathered}
$$

Equation (21) shows net moment results from the canard asymmetry since the angles of attack on each canard are different. This means that, during flight, the canards may contribute to transient coning motion when $\left|M_{C}\right|$ is sufficiently large.

\section{Extended Projectile Linear Theory}

The usual assumptions corresponding to projectile linear theory are products formed by transverse components of $C_{B}\left(\mathbf{V}_{\otimes / I}\right)$,
$C_{B}\left(\mathbf{V}_{C j / I}\right), C_{B}\left(\omega_{B / I}\right)$, and $\delta$ are negligible, plus axial components $\mathbf{I}_{B} \bullet C_{B}\left(\mathbf{V}_{\otimes / I}\right)=V$ and $\mathbf{I}_{B} \bullet C_{B}\left(\boldsymbol{\omega}_{B / I}\right)=p$ are constant. These assumptions lead to a fourth-order initial value system with constant coefficients and time-dependent driving terms:

$$
\begin{aligned}
& \left\{\begin{array}{c}
\dot{v} \\
\dot{w} \\
\dot{q} \\
\dot{r}
\end{array}\right\}=\left[\begin{array}{cccc}
-\frac{A V}{D} & p & 0 & -V \\
-p & \frac{V\left(V_{2}-A\right)}{D} & V\left(V_{3}+1\right) & 0 \\
\frac{B V}{D^{2}} & \frac{\left(M_{2}+C\right) V}{D^{2}} & \frac{\left(M_{3}+E\right) V}{D} & -\frac{F V-p D}{D} \\
-\frac{C V}{D^{2}} & \frac{B V}{D^{2}} & \frac{F V-p D}{D} & \frac{E V}{D}
\end{array}\right]\left\{\begin{array}{l}
v \\
w \\
\sin (p t) \frac{g}{D} \\
q \\
r
\end{array}\right\} \\
& =\left\{\begin{array}{c}
\cos (p t) \frac{g}{D}-\frac{\pi \rho D^{2} V^{2} C n a c}{4 m} \delta \\
\frac{\pi \rho \Delta x D^{2} V^{2} C n a c}{4 I_{T}} \delta \\
\frac{\pi \rho \Delta y c^{2} p D^{2} V \text { Cnac }}{4 I_{T}} \delta
\end{array}\right\}
\end{aligned}
$$

The full expressions for the matrix are lengthy, and they are provided in the Appendix, which also shows the contributions due to asymmetry are $\left(V_{2}, V_{3}, M_{2}, M_{3}\right)$. When these contributions are equal to zero, no asymmetry exists, and the system in Eq. (22) returns to the familiar linear theory describing symmetric projectiles written in the body frame. The eigenvalues of the system above split into two conjugate pairs: a fast mode and a slow mode denoted as $S_{F}$ and $S_{S}$ $\left(S_{F, S}=\lambda_{F, S} \pm i \dot{\phi}_{F, S}\right)$, respectively. Dynamic stability of Eq. (22) is determined by the real parts of these eigenvalues. The linear combination of damped and undamped sinusoid terms forming the solution of Eq. (22) are obtained using the Laplace transform followed with employing the Mellin inverse formula [18]. The particular canard time-dependent deflection angle $\delta$ used to generate in this report has the form

$$
\delta=\Delta \sin (k t), \quad \Delta=10^{\circ}
$$

\section{Model Validation}

To establish the utility of the extended projectile linear theory developed above, time domain solutions from the linear model [Eq. (16)] are compared with time domain calculations of the nonlinear model [Eqs. (4-7)]. The particular example given here has mass properties $m=1 . \overline{2} 6 \overline{8} 0 \mathrm{slug}$, moments of inertia $I_{X}=2.449 \times$ $10^{-2}$ slug $\mathrm{ft}^{2}$ and $I_{T}=0.51569 \mathrm{slug} \cdot \mathrm{ft}^{2}$, and air density $\rho=$ $2.38 \times 10^{-3} \mathrm{slug} \cdot \mathrm{ft}^{-3}$. The spin rate $p=75.4 \mathrm{~s}^{-1}$, and the forward velocity $V=558.0 \mathrm{ft} \cdot \mathrm{s}^{-1}$. This projectile's geometry characteristics are $D=0.343521 \mathrm{ft}, \mathrm{SL}_{\mathrm{COP}}=0.7284 \mathrm{ft}, \mathrm{SL}_{\mathrm{MAG}}=0.2390 \mathrm{ft}$, $S_{L C G}=1.17787 \mathrm{ft}, \Delta x c=1.2434 \mathrm{ft}$, and $\Delta y c=0.134 \mathrm{ft}$. The projectile aerocoefficients are $C_{N A}=5.8150, C_{N A C}=0.4875$, $C_{Y P A}=-28$, and $C_{M Q}=-151.91$, and all reference areas are based on diameter $D$.

Comparisons of linear theory to nonlinear 6-DOF calculations, along with data from flight experiments, are shown in Figs. 3-6. Figure 3 compares linear theory to 6-DOF canard moment ratios $M_{C Y} / \bar{M}_{C Y_{\mathrm{MAX}}}$ as functions of time for $5.0 \mathrm{~s}<t<8.0 \mathrm{~s}$. The scale

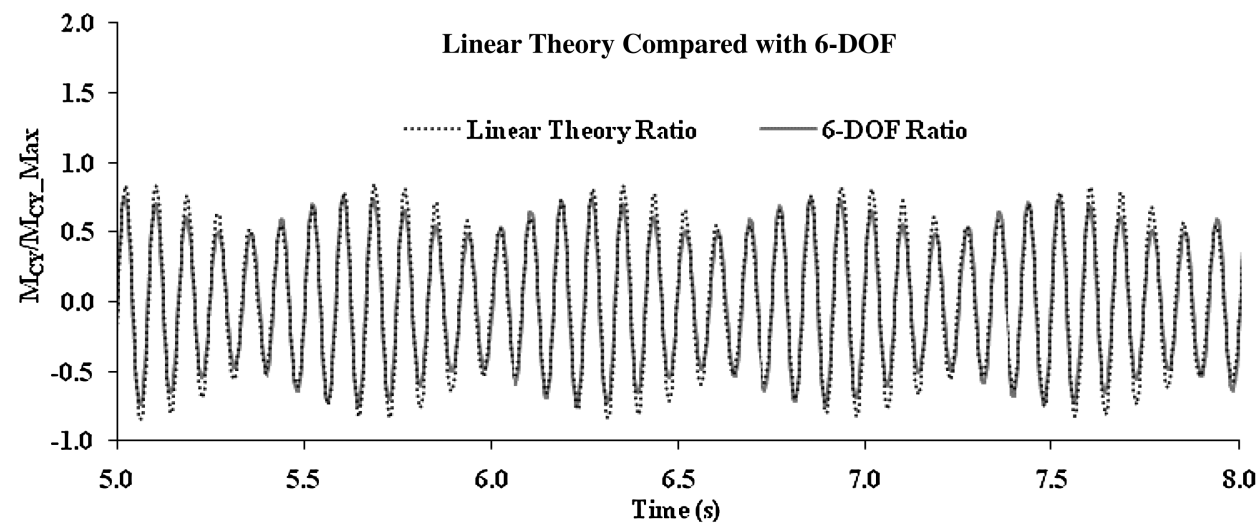

Fig. 3 Comparison of $M_{C Y} / M_{C Y_{\mathrm{MAX}}}$ vs time. 


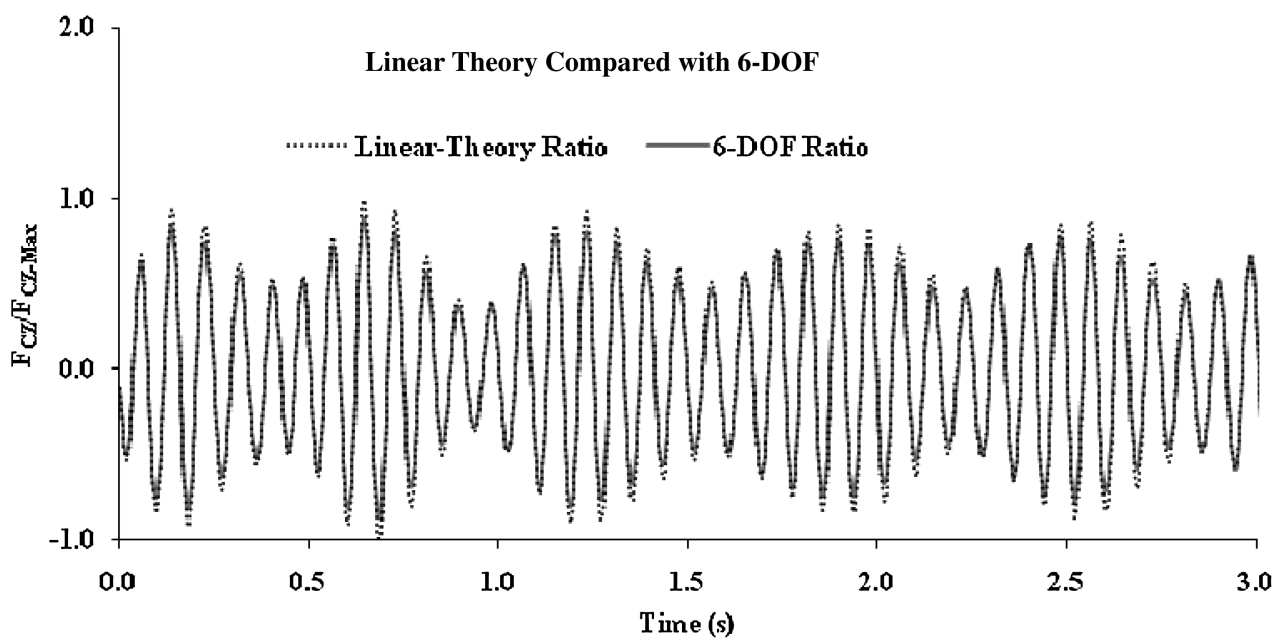

Fig. 4 Comparison of $\boldsymbol{F}_{C Z} / \boldsymbol{F}_{C Z_{\mathrm{MAX}}}$ vs time.

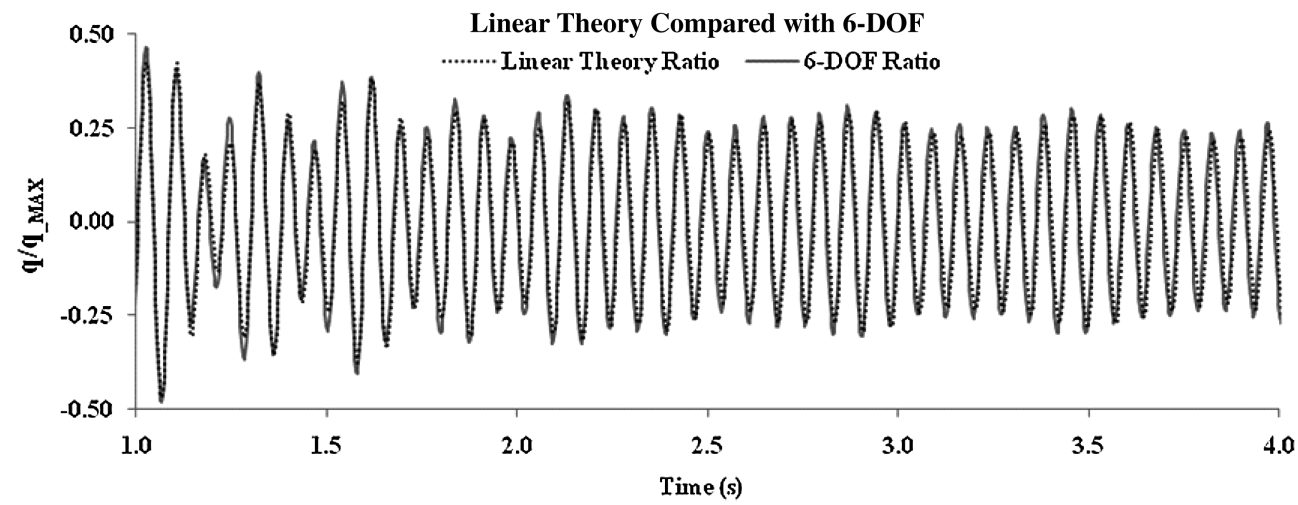

Fig. 5 Comparison of $q / q_{\mathrm{MAX}}$ vs time.

factor $M_{C Y_{\mathrm{MAX}}}$ is the maximum value of $M_{C Y}$ of the linear theory calculations of the duration of the flight $0 \mathrm{~s}<t<30 \mathrm{~s}$, for which the canard activation frequency is chosen as $k=p$ to effectively maximize projectile range (glide).

A similar chart showing the canard force ratio $F_{C Z} / F_{C Z_{\mathrm{MAX}}}$ is given in Fig. 4 , and the scale factor $F_{C Z_{\mathrm{MAX}}}$ is the maximum value of the linear $F_{C Z}$ over the 30 s flight.

The variables in Figs. 3 and 4 are chosen for comparison since the nonlinear effects due to the 6-DOF are most dissimilar for the canard force and moment. A chart comparing linear and 6-DOF calculations of the similarly scaled $q / q_{\mathrm{MAX}}$ ratios is shown in Fig. $\underline{5}$

One way to view the angular motion of this asymmetric projectile is to consider the time dependence of $\alpha, \beta$, defined as follows [3]:

$$
\left\{\begin{array}{l}
\alpha \\
\beta
\end{array}\right\} \equiv\left\{\begin{array}{c}
\tan ^{-1}\left(\frac{\cos (p t) v-\sin (p t) w}{V}\right) \\
\tan ^{-1}\left(\frac{\cos (p t) w+\sin (p t) v}{V}\right)
\end{array}\right\}
$$

Figure 6 has a chart for flight times $t \leq 30 \mathrm{~s}$ comparing linear theory and 6-DOF calculations along with flight-test data of the projectile modeled in the above calculations [19].

Evidently, linear theory captures most of the physics governing the angular motion of this projectile when subjected to two activating canards configured at $\phi=0$ and $\phi=\pi$. The magnitude of transitory coning behavior for $t \leq 30 \mathrm{~s}$ is caused by the canard moments $M_{C}$ and the body moment $B$. Essentially, the canards induce the angle of attack in the body. At the angle of attack, the Magnus moment of the body contributes to the transient coning along with the canard actuation. In the plot of Fig. 6, the Magnus moment appears as nearly circular orbits that complete a full revolution at the projectile yaw rate. The perturbations in the orbits of Fig. $\underline{6}$ are due to the faster canard actuation frequency. The canard actuation-induced angle of attack is near $3^{\circ}$, as seen in Fig. $\underline{6}$. The experimental and linear theory-based transient coning magnitude is around $3^{\circ}$, and the angular motion perturbations due to canard actuation at the roll rate are evident in Fig. 6.

The steady-state coning motion corresponding to the particular solution of Eq. (22) is attained for flight times $\geq 200 \mathrm{~s}$ due to the size of $\left|\lambda_{F, S}\right|$. This steady-state motion is an offset ellipse centered at the

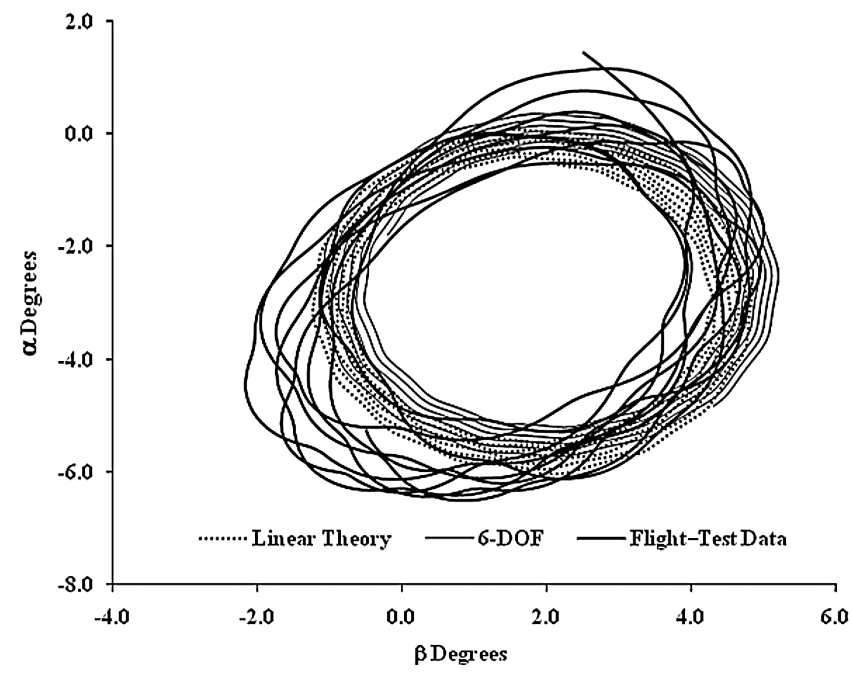

Fig. 6 Comparisons of transient coning motions for time $\leq 30 \mathrm{~s}$. 


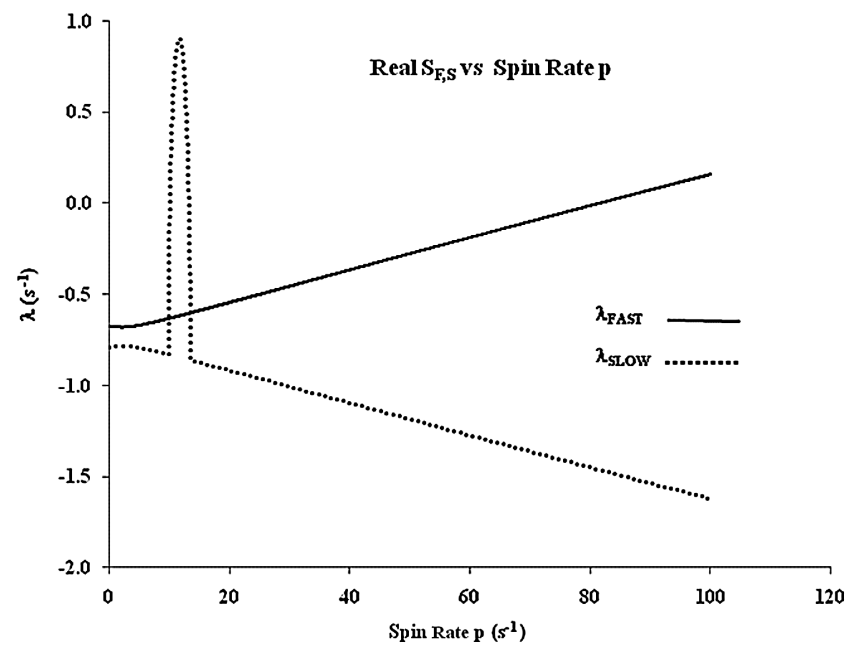

Fig. 7 Fast and slow real roots $\lambda_{\text {FAST,SLOW }}$ as functions of spin rate $p$.

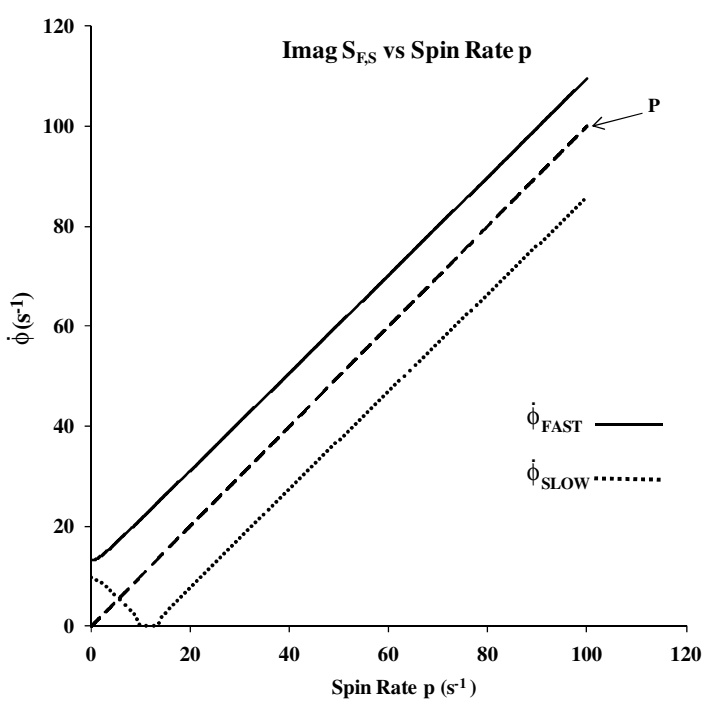

Fig. 8 Fast and slow real roots $\phi_{\text {FAST,SLOW }}$ as functions of spin rate $p$.

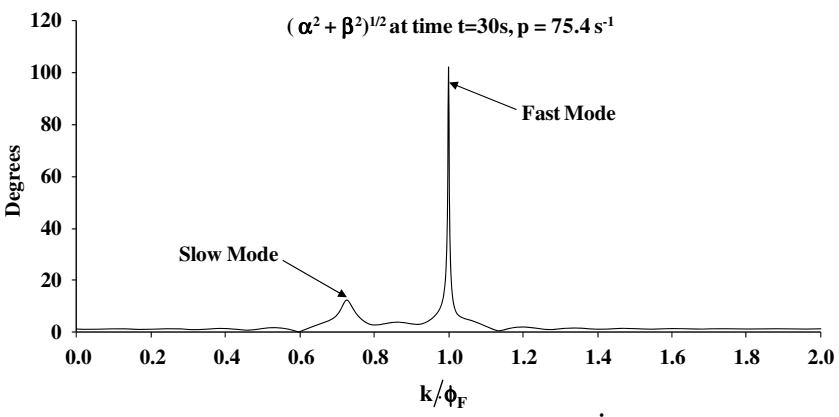

Fig. 10 Metric resonance vs $k / \dot{\phi}_{F}$.

geometric center of Fig. $\underline{6}$ with vanishing small radii (analysis not presented here since the expressions are judged to be too long).

\section{Stability Analysis}

Numerical values of fast and slow eigenvalues $S_{F, S}$ are presented as functions of projectile spin rate $p$, given in Figs. 7 and 8. The slow mode $S_{S}$ is real and positive for $10.2 \mathrm{~s}^{-1} \leq p \leq 13.3 \mathrm{~s}^{-1}$, which indicates dynamic instability; see Fig. 5 of [3]. As $p$ increases, $p \geq 79.0 \mathrm{~s}^{-1}$, causes the fast mode contribution to grow unstable.

Another way to present the numerical values of fast and slow eigenvalues $S_{F, S}$ is to form a root locus plot for changing projectile spin rate $p$, shown in Fig. 9 .

These eigenvalues provide a tool for addressing dynamic stability of the asymmetric canard configuration modeled in this effort.

Sinusoidal driving terms representing canard actuation [Eq. (23)] cause component solutions of Eq. (23) to have terms with form

$$
\frac{G(t)}{\left(\dot{\phi}_{F, S} \pm k\right)^{2}+\lambda_{F, S}^{2}}
$$

$G(t)$ represents a time-dependent numerator

Thus, in the event $\left|\lambda_{F, S}\right| \ll 1, k \rightarrow\left|\dot{\phi}_{F, S}\right|$ may potentially generate flight instability when the canards are actuated as sinusoids. A metric to demonstrate this potential problem is defined in Eq. (26):

$$
\operatorname{Max} \sqrt{\alpha^{2}+\beta^{2}} \quad \forall t \in(0,30 \mathrm{~s})
$$

Figure 10 shows a plot of this behavior where the spin rate is held constant $\bar{p}=75.4 \mathrm{~s}^{-1}$ for varying values of $k$.

Apparently, when the frequency $k$ is in the neighborhood of the fast mode, the frequency $\dot{\phi}_{F}$ causes the strongest destabilizing effect.

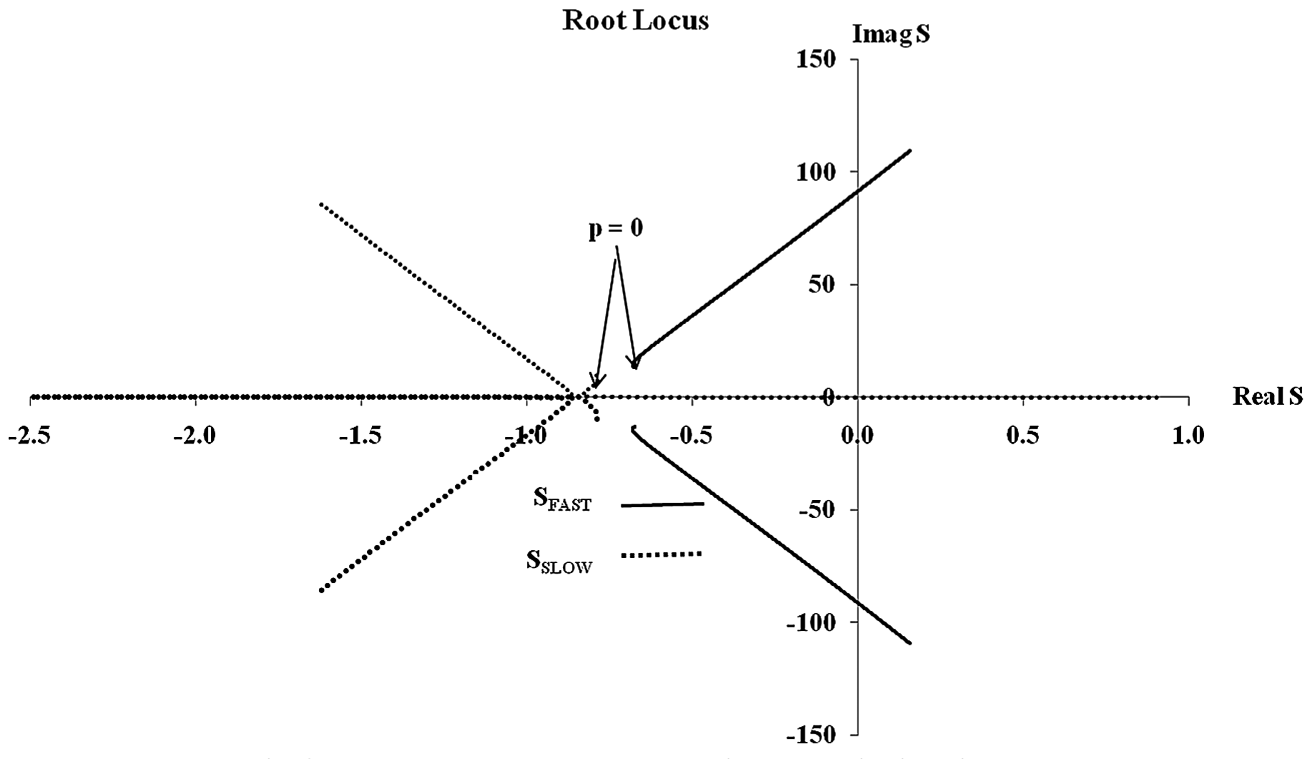

Fig. 9 Fast and slow roots $S$ parameterized by projectile spin rate $p$. 


\section{Conclusions}

An extension of the familiar linear-projectile theory for symmetric and asymmetric projectiles has been formulated and solved. This extension accounts for configuration asymmetries caused by two actuating canards placed at roll angles $\phi=0$ and $\phi=\pi$. The documentation shows that a wide class of configuration asymmetries can be addressed by the techniques given in this report. One of the results obtained in this paper presents a way to analyze the dynamic stability of projectiles exhibiting asymmetries. This can have an important impact on the design of projectiles. The importance of canard actuation focuses on a potential problem causing flight instability when the actuation frequencies are too close to one of the modal yawing frequencies. A spinning projectile with two canards activated as sinusoids was shown to contribute to transient coning motion when this additional moment was sufficiently large. The closed-form solutions discussed in this report were shown to compare well with standard 6-DOF calculations. Postprocessing solutions given here to obtain all state components of asymmetric projectiles may prove useful in developing firmware. The asymmetric linear theory reduces to the familiar linear symmetric theory when the asymmetries vanish.

\section{Appendix}

$$
\begin{gathered}
A=\frac{\pi \rho D^{3} C_{N A}}{8 \mathrm{~m}} \quad B=\frac{\pi \rho p D^{5} C_{\mathrm{YPA}}\left(\mathrm{SL}_{\mathrm{MAG}}-\mathrm{SL}_{\mathrm{CG}}\right)}{16 I_{T} V} \\
C=\frac{\pi \rho D^{4} C_{N A}\left(\mathrm{SL}_{\mathrm{COP}}-\mathrm{SL}_{\mathrm{CG}}\right)}{8 I_{T}} \quad E=\frac{\pi \rho D^{5} C_{M Q}}{16 I_{T}} \\
F=\frac{p D I_{x}}{I_{T} V} \quad V_{2}=-\frac{\pi \rho D^{3} C_{N A C}}{4 m} \\
M_{2}=\frac{\pi \rho D^{4} \Delta x c C_{N A C}}{4 I_{T}} \quad M_{3}=-\frac{\pi \rho D^{2} \Delta x c C_{N A C}}{4 m} \\
4 I_{T}
\end{gathered}
$$

\section{References}

[1] Fresconi, F., and Plostins, P., "Control Mechanism Strategies for SpinStabilized Projectiles," Journal of Aerospace Engineering, Vol. 224, No. 9 2010, pp. 979-991. doi:10.1243/09544100JAERO705

[2] Murphy, C. H., "Symmetric Missile Dynamic Instabilities: A Survey," 18th AIAA Aerospace Sciences Meeting, AIAA Paper 1980-0320, Jan 1980.

[3] Charles H. Murphy, "Angular Motion of Spinning Almost Symmetric Missiles," Journal of Guidance, Control, and Dynamics, Vol. 2, No. 6, Nov.-Dec. 1979, pp. 504-510. doi: $10.2514 / 3.55916$

[4] Hodapp, A. E., "Effect of Mass Asymmetry on Ballistic Match of Projectiles," Journal of Spacecraft and Rockets, Vol. 13, No. 12, 1976, pp. $757-760$. doi: $10.2514 / 3.57137$

[5] Weber, D. J., "Simplified Method for Evaluating the Flight Stability of Liquid-Filled Projectiles," Journal of Spacecraft and Rockets, Vol. 31, No. 1, 1994, pp. 130-134. doi: $10.2514 / 3.26412$

[6] Murphy, C. H., "Angular Motion of a Spinning Projectile with a Viscous Liquid Payload," Journal of Guidance, Control, and Dynamics, Vol. 6 , No. 4, July-Aug. 1983 , pp. 280-286. doi: $10.2514 / 3.19830$

[7] Murphy, C. H., "Influence of Moving Internal Parts on the Angular Motion of Spinning Projectiles," Journal of Guidance, Control, and Dynamics, Vol. 1, No. 2, March-April 1978, pp. 117-122. doi: $10.2514 / 3.55753$

[8] Cobb, K. K., Whyte, R. H., and Laird, P. K., "The Effects of a Moving Components on the Motion of a 20-mm Projectile," 11th AIAA Aerodynamics Testing Conference, New York, AIAA Paper 19800428,1980

[9] Hodapp, A. E., "Passive Means for Stabilizing Projectiles with Partially Restrained Internal Members," Journal of Guidance, Control, and Dynamics, Vol. 12, No. 2, 1989, pp. 135-139. doi: $10.2514 / 3.20382$

[10] Soper, W. G., "Projectile Instability Produced by Internal Friction," AIAA Journal, Vol. 16, No. 1, 1978, pp. 1-11. doi: $10.2514 / 3.60848$

[11] Costello, M., and Peterson, A., "Linear Theory of a Dual-Spin Projectile in Atmospheric Flight," Journal of Guidance, Control, and Dynamics, Vol. 23, No. 5, 2000, pp. 789-797. doi: $10.2514 / 2.4639$

[12] Cooper, G., "Extending the Jump Analysis for Aerodynamic Asymmetry," Journal of Spacecraft and Rockets, Vol. 43, No. 3, May-June 2006, pp. 700-703. doi:10.2514/1.17718

[13] Murphy, C. H., "Instability of Controlled Projectiles in Ascending or Descending Flight," Journal of Guidance, Control, and Dynamics, Vol. 4, No. 1, 1981, pp. 66-69. doi: $10.2514 / 3.19716$

[14] Cooper, G., "Influence of Yaw Cards on the Yaw Growth of Spin Stabilized Projectiles," Journal of Aircraft, Vol. 38, No. 2, 2001, pp. 266-270. doi: $10.2514 / 2.2784$

[15] Guidos, B., and Cooper, G., "Closed Form Solution of Finned Projectile Motion Subjected to Simple In-Flight Lateral Impulse," AIAA Paper 2000-0767, 2000.

[16] Burchett, B., Peterson, A., and Costello, M., "Prediction of Serving Motion of a Dual-Spin Projectile with Lateral Pulse Jets in Atmospheric Flight," Mathematical and Computer Modelling, Vol. 35, Nos. 1-2, 2002, pp. 1-14.

doi:10.1016/S0895-7177(01)00143-1

[17] Pechier, M., Guillen, P., and Cayzac, R., "Magnus Effect over Finned Projectiles," Journal of Spacecraft and Rockets, Vol. 38, No. 4, JulyAug. 2001, pp. 542-549. doi: $10.2514 / 2.3714$

[18] Carrier, G. F., Krook, M., and Pearson, C. E., Functions of a Complex Variable: Theory and Technique, Hod Books, Ithaca, NY, 1983, pp. 364-365.

[19] Fresconi, F., and Harkins, T., "Aerodynamic Characterizations of Asymmetric and Maneuvering $105 \mathrm{~mm}, 120 \mathrm{~mm}$, and $155 \mathrm{~mm}$ FinStabilized Projectiles Derived from Telemetry Experiments," AIAA Atmospheric Flight Mechanics Conference, AIAA, Paper 2011-6268, Aug. 2011. 